Research Article

\title{
Support Vector Machine-Based Analysis of Holmium Laser Lithotripsy in Treatment of Urinary Calculus from Ultrasound Images
}

\author{
Hu Li $\mathbb{D}^{1},{ }^{1}$ Zhijun Chen $\mathbb{D}^{2},{ }^{2}$ Dezhi Kong $\mathbb{D}^{1},{ }^{1}$ Zhiqiang Huang $\mathbb{D}^{1},{ }^{1}$ and Ningning Wang $\mathbb{D}^{1}$ \\ ${ }^{1}$ Department of Urology, General Hospital of Wanbei Coal-Electric Group, Suzhou 234000, Anhui, China \\ ${ }^{2}$ Department of Urology, The First Affiliated Hospital of Bengbu Medical College, Bengbu 233000, Anhui, China \\ Correspondence should be addressed to Zhijun Chen; 51082@bbmc.edu.cn
}

Received 30 September 2021; Revised 5 November 2021; Accepted 8 November 2021; Published 7 December 2021

Academic Editor: M Pallikonda Rajasekaran

Copyright (c) $2021 \mathrm{Hu} \mathrm{Li}$ et al. This is an open access article distributed under the Creative Commons Attribution License, which permits unrestricted use, distribution, and reproduction in any medium, provided the original work is properly cited.

\begin{abstract}
This study was to investigate the application value of ultrasound images optimized by support vector machine (SVM) algorithm in the efficacy analysis of holmium laser lithotripsy in the treatment of urinary calculi. 92 patients for treatment of UC were selected as research subjects, with 46 cases in each group. The control group received pneumatic lithotripsy for diagnosis and treatment. The observation group received holmium laser lithotripsy for calculus treatment. The perimeter and area of the defect and the length and width of the external distance of the most effective area of the defect were used as classification features, and a classifier based on SVM was constructed and applied to it. After treatment, the success rate, operation duration, stone clearance time, and hospital stay of the two groups were comprehensively evaluated. The results showed that the success rate of the observation group adopting holmium laser lithotripsy was 100\%. The duration of operation in the observation group was $(29.7 \pm 7.65) \mathrm{min}$, and the time to clear calculus was $(6.99 \pm 5.29)$ days. The length of hospital stay was $(3.67 \pm 2.9)$ days. The probability of complications in the observation group was $3.11 \%$. The observation group was superior to the control group in all surgical indicators $(95 \%, 40.7 \pm 8.36$ minutes, $14.1 \pm 7.21$ days, and $5.12 \pm 3.72$ days), with considerable differences between groups $(P<0.05)$. The strong support structure similarity information improved the detection and diagnostic analysis ability of ultrasonic images. In conclusion, after ultrasound image evaluation based on SVM algorithm, the adoption of holmium laser lithotripsy can effectively improve the success rate of patients with urinary system disease, which was worthy of clinical adoption and promotion.
\end{abstract}

\section{Introduction}

With the continuous acceleration of the modern process, various environmental pollution and life and work pressure will cause health problems to people. Urinary calculus has become a common urinary system disease that threatens people's health. Urinary calculus is one of the most common diseases of the urinary system [1], whose main symptoms are hematuria, dysuria, pain, frequent urination, urgency, and painful urination. When urinary problems occur, routine urine tests as well as color Doppler ultrasound, CT, or MRI are needed to confirm the diagnosis, and the appropriate treatment is selected after the diagnosis [2]. According to the current survey results in China, the number of patients suffering from urinary system disease in China is increasing year by year, which affects the life, work, and study of patients to a certain extent [3]. Moreover, the disease is currently being transferred from elderly patients to young adults [4]. Related research suggested that urinary system disease was more likely to occur in men. The incidence rate of male to female reached $5: 1$. The incidence of calculus in the upper urinary tract such as ureter and kidney showed an upward trend, and the incidence of calculus in the lower urinary tract such as the bladder showed a downward trend [5]. If the UC cannot be effectively treated, it will cause great damage to the human kidney function, and it may cause uremia in the later stage [6]. In some patients with severe renal calculus, the kidney tissue was found to be replaced by 
fat and even disappeared completely. If renal calculus patients are not treated for a long time, they will eventually develop into renal failure, endangering the patient's life.

In recent years, there are many ways to treat urinary system disease. Extracorporeal shock wave lithotripsy, traditional Chinese medicine, and surgical treatment are all common ones. Surgical treatment is an effective method for clinical treatment of urinary calculi in China. Early surgical techniques were low, and open surgery was mostly used. With the continuous progress of surgical techniques, the surgical treatment of urinary calculi has become minimally invasive. At present, titanium laser lithotripsy is a laser lithotripsy used in the treatment of urinary calculi in China [7].

In the process of examining the patient's urinary system, the classification of ultrasound images has always been a problem studied and discussed by medical experts and scholars. How to quickly and effectively screen the available images in the process of identifying ultrasound images is the research focus. Problems such as the unclear boundaries of the pathological characteristics and the variety of samples will affect the doctor's diagnosis and treatment of patients, which will also delay the best treatment time for patients. In response to these problems, the adoption of genetic algorithms to optimize the ultrasound image classification method of support vector machine (SVM) can solve the above problems to a certain extent, improve the comprehensive recognition rate, and effectively assist medical staff to classify and recognize ultrasound image defects. Technicians utilize ultrasonic nondestructive testing technology to detect defects $[8,9]$, which is effective, fast, and convenient to identify defects in ultrasound images. It is of great significance to reduce the error of manual identification for the manufacture of metal workpiece. In solving the problem of small sample, nonlinear, and highdimensional pattern recognition, SVM has great advantages $[10,11]$. In this study, 92 patients were selected as the research objects, and the genetic algorithm was used to optimize the SVM to obtain an effective classifier. The ultrasonic image defects of urinary system diseases were classified and diagnosed to analyze the application of ultrasonic images based on SVM algorithm in the treatment of urinary calculi by holmium laser lithotripsy and provide certain reference for the clinical treatment of urinary calculi.

\section{Materials and Methods}

2.1. Selection of Research Sample. 92 patients who came to the hospital for treatment of UC from July 2016 to July 2018 were selected as research subjects. 46 patients in the control group were diagnosed and treated with pneumatic lithotripsy. 46 patients in the observation group adopted holmium laser lithotripsy for calculus treatment. Then, the efficacy of the two groups was observed. The research had been approved by the medical ethics committee of the hospital, and the patients and their family members also understood the specific situation of the research and had signed inform consent forms.

Inclusion criteria: i) patient's age range was 20-65 years old; ii) urolithiasis was diagnosed by ultrasound; iii) patients were with no complication of cardiovascular diseases and liver and kidney dysfunction.

Exclusion criteria were as follows: patients with severe cardiopulmonary disease or liver and kidney dysfunction; patients with diabetes, chronic nephritis, or hypertension; patients with urinary system infection, severe renal hydrops, urethral stricture, or staghorn-shaped calculus; and patients with other serious systemic diseases.

2.2. Support Vector Machine Classifier. The basic principle of the SVM algorithm is finding a hyperplane for a given sample set, and this hyperplane can correctly classify the unseen test set into two categories. The perimeter and area of the ultrasound image defect and the length and width of the circumscribed distance of the most effective area of the defect are deemed as classification features, and $x_{1}$ is a vector containing four kinds of data information [12].

The classification hyperplane of ultrasonic image defects is expressed as follows:

$$
f(z)=\alpha^{1} z+b=0,
$$

where $\alpha^{1}$ is the normal vector of the hyperplane and $b$ is the translation distance of the hyperplane. Since the edges and corners of the ultrasound image are not clear and the discrimination is poor, the distance between the sample and the hyperplane should be as large as possible. To reduce the human error, slack variables need to be added to the objective function, so the objective function can be expressed as follows:

$$
\begin{aligned}
& \min \frac{1}{2}\|\alpha\|^{2}+A \sum_{r=1}^{i} \omega_{1}, \\
& t . f, h\left(\alpha^{1} z_{r}+b\right) \geq+1, \\
& \omega_{1} \geq 0, \quad r=1,2 \ldots, n .
\end{aligned}
$$

where $A$ represents the error cost coefficient, which will affect the accuracy of ultrasound image defect classification and the fault tolerance to noise, and $\omega_{1}$ is the slack variable corresponding to the data point $z_{r}$. When facing the problem of nonlinear segmentation, the SVM algorithm needs to transform the variables into a high-dimensional space to solve them. The kernel function appears to solve the dimensional explosion problem, which is represented by $M\left(s_{r}, s\right)$. Any function that satisfies the theorem can be regarded as a kernel function. In this work, the Gaussian meridional basis kernel function is adopted.

$$
M\left(s_{r}, s\right)=\exp \left(-\frac{\left(s, s_{r}\right)^{2}}{\delta^{2}}\right),
$$

where $\delta$ is the mapping that affects the sample space to the feature space. The generalized Grange multiplier is introduced to the objective function again, and when the AAT condition is met, the objective function can be transformed into the following equation: 


$$
\begin{aligned}
& \max \sum_{r=1}^{i} c_{1}-\frac{1}{2} \sum_{r=1}^{n} \sum_{k=1}^{n} c_{m} c_{k} h_{m} h_{k} M\left(s_{r}, s\right) \\
& t . f \sum_{r=1}^{n} c_{m} h_{m}=0 \\
& 0 \leq c_{1} \leq C, \quad r=1,2 \ldots, n,
\end{aligned}
$$

where $c_{1}$ is the Lagrange coefficient; then, the score function is shown in the following equation:

$$
f(z)=\sum_{r=1}^{n} c_{m} h_{m}\left(s_{r}, s\right)+b .
$$

Based on the SVM algorithm, the problem can be searched and solved globally without falling into a local optimal solution. The inherent parallelism in the calculation process can improve the solution speed. Figure 1 shows the process of genetic algorithm optimization based on support vector machine algorithm.

2.3. Surgical Methods. In the two groups of patients, patients in the observation group were treated with titanium laser lithotripsy. AGCO Kaineng 30W holmium laser made in China was used, and the cystoscope sheath, tube, and urethroscope were placed through the urethral opening of the patient under anesthesia. Under anesthesia, the cystoscope sheath, tubescope, and urethroscope were inserted into the body from the urethral opening. Then, the brightness, light source system, and focal length were adjusted. The adsorption pump and the attention pump were opened, and the ureteroscope water filling connection pipe was connected [13]. After the position of calculus was determined, the laser fiber was inserted and observed under the uretero-nephroscope. The fiber diameter range was 550-600 $\mu \mathrm{m}$. When the fiber head reached calculus, the power would be adjusted to $0.8-1.2 \mathrm{~J} /(5-10 \mathrm{~Hz})$. Under the condition of direct vision, the small calculus powder smaller than $3 \mathrm{~mm}$ was washed and crushed by manual continuous pulse and then taken out. If there were hyperplastic polyps at the calculus site, holmium laser ablation was required first, and then patients should stay in the hospital for observation for about a week [14].

Patients in the control group were treated with pneumatic lithotripsy. Epidural anesthesia was performed on patients. After successful anesthesia, vital signs of patients were observed, lithotomy position was taken, and ureteroscope was inserted into the bladder through urethra. Then, the ureteroscope was slowly placed to the diseased ureter side along the guide wire. After the stone was fully exposed, the lithotripsy needle can be inserted at the operation port of ureteroscope, so that it can fully contact the stone site, and then the pressure was gradually increased. After starting the pressure, the lithotripsy operation can be performed. Smaller stones can be discharged by themselves. Larger stones were removed by baskets. After the operation, the patency of the ureter should be carefully examined, and finally double J tube and urethral catheter should be placed.

2.4. Evaluation Indexes. The operation time, calculus clearance, and intraoperative blood loss of the two groups of patients were compared, so were the length of stay of the two groups of patients, the time of indwelling catheter, and the occurrence of complications after the operation. Then, the clinical effects of different treatments could be compared.

2.5. Statistical Methods. Data processing was analyzed by SPSS19.0 version statistical software. Measurement data were expressed by mean \pm standard deviation. Counting data were expressed by percentage (\%). One-way analysis of variance was employed for pairwise comparison of running time. The comparison of age, height, weight, course of disease, ratio of male to female, and diagnostic accuracy between groups was performed by paired $t$-test. The difference was statistically significant at $P<0.05$.

\section{Results}

3.1. Basic Information of Patients. Among 92 patients with urinary system disease, 67 were males and 25 were females. The average age of 92 patients was $(53.3 \pm 4.01)$ years. There were 31 males and 15 females in the control group. The average height of males was $(177.4 \pm 7.2) \mathrm{cm}$, and the average height of females was $(160.8 \pm 6.08) \mathrm{cm}$. The average weight of females was $(51.8 \pm 9.1) \mathrm{kg}$, and the average weight of males was $(69.3 \pm 9.7)$ $\mathrm{kg}$. The average age of males was $(49.2 \pm 6.1)$ years and the average age of females was $(47.1 \pm 4.3)$ years (Figure 2$)$. There were 36 male patients and 10 female patients in the observation group. The average height of males was $(171.3 \pm 9.4) \mathrm{cm}$, and the average height of females was $(159.4 \pm 7.8) \mathrm{cm}$. The average weight of females was $(52.9 .8 \pm 7.5) \mathrm{kg}$, and the average weight of males was $(70.3 \pm 7.7) \mathrm{kg}$. The average age of females was $(49.8 \pm 5.1)$ years, and the average age of males was $(52.7 \pm 7.9)$ years (Figures 3 and 4 ).

From Table 1, the calculus position of most patients was on the left side, the left-side diameter of calculus was $(2.09 \pm 1.23) \mathrm{cm}$, and the right-side diameter was $(2.39 \pm 0.57) \mathrm{cm}$.

According to Table 1, there were no statistically significant differences in age, height, weight, or male and female ratio between the control group and the observation group $(\mathrm{P}>0.05)$.

Figures 5 and 6 show ultrasound images of patients with typical urinary system diseases. Figure 5 shows a male patient (47 years old); Figure 6 shows a female patient ( 49 years old). Both were diagnosed with ureteral calculi by Doppler ultrasonography.

3.2. Comparison of Intraoperative and Postoperative Indicators between the Two Groups of Patients. The number of successful operations in the observation group of 46 patients was 46 , and the success rate reached $100 \%$. In the 


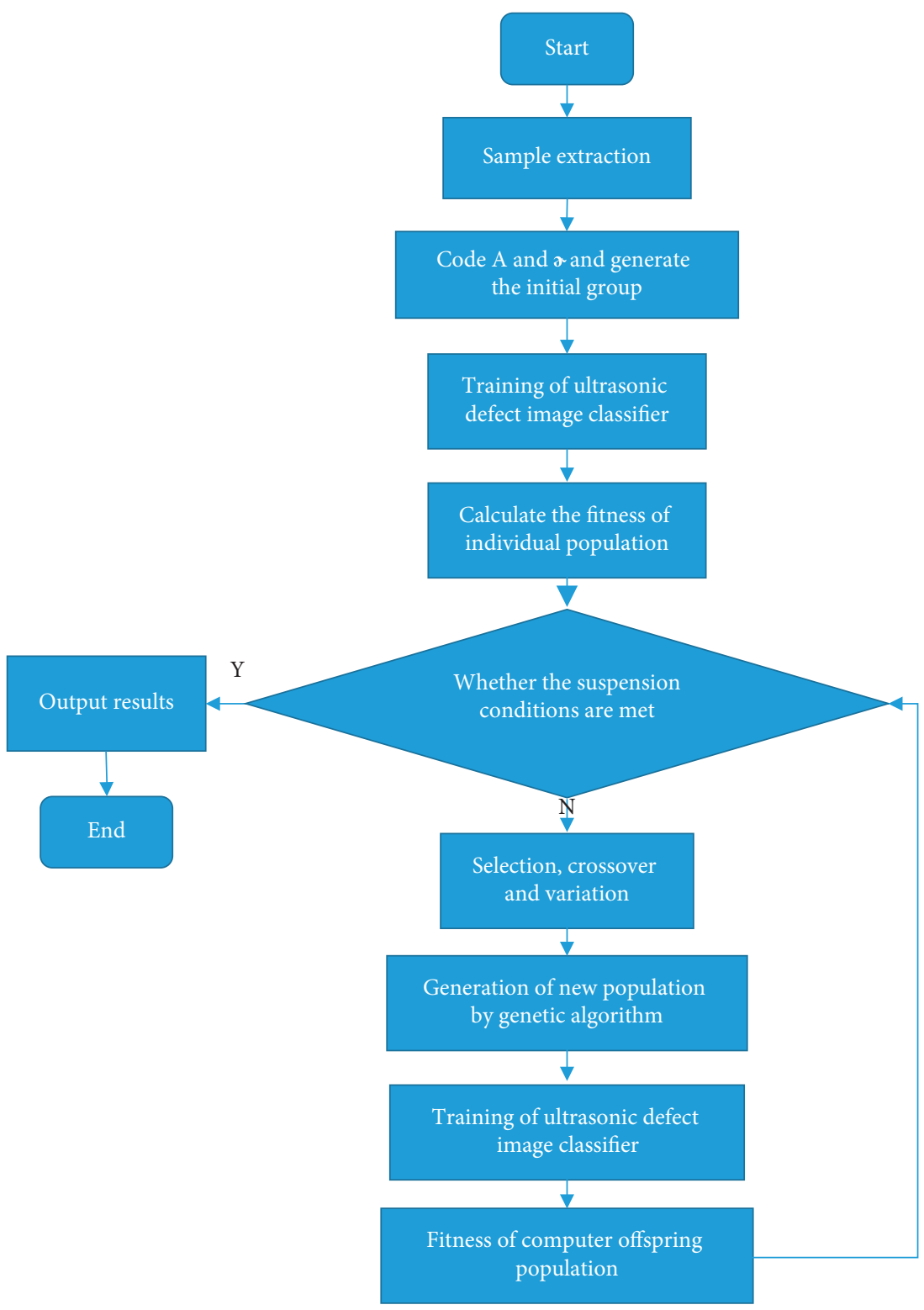

FIGURE 1: The algorithm flow of genetic algorithm optimization SVM.

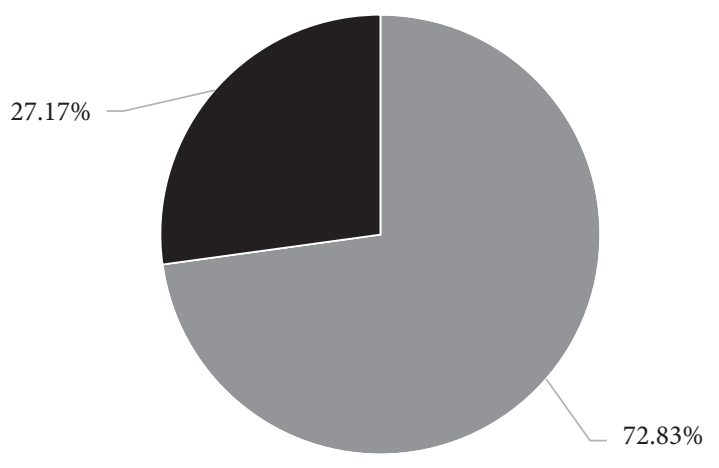

- Men

- Women

FIGURE 2: Distribution of the ratio of male to female patients.

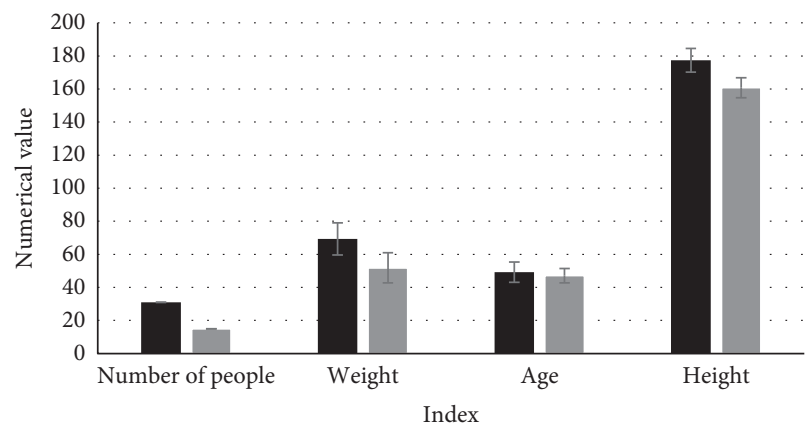

- Men

- Women

Figure 3: Basic indexes of patients in the control group. 


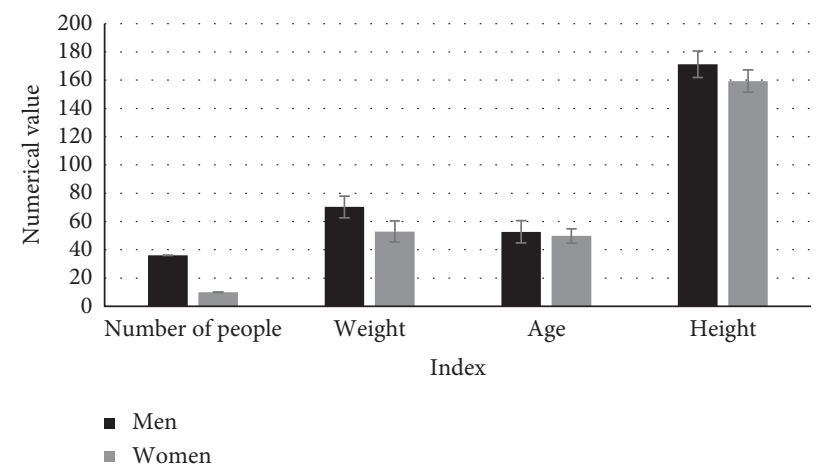

FigURE 4: Basic indexes of patients in the observation group.

TABLE 1: Basic pathological conditions of patients.

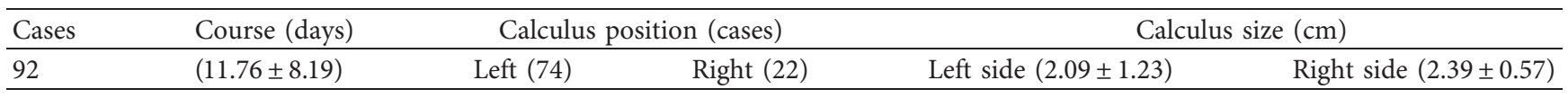
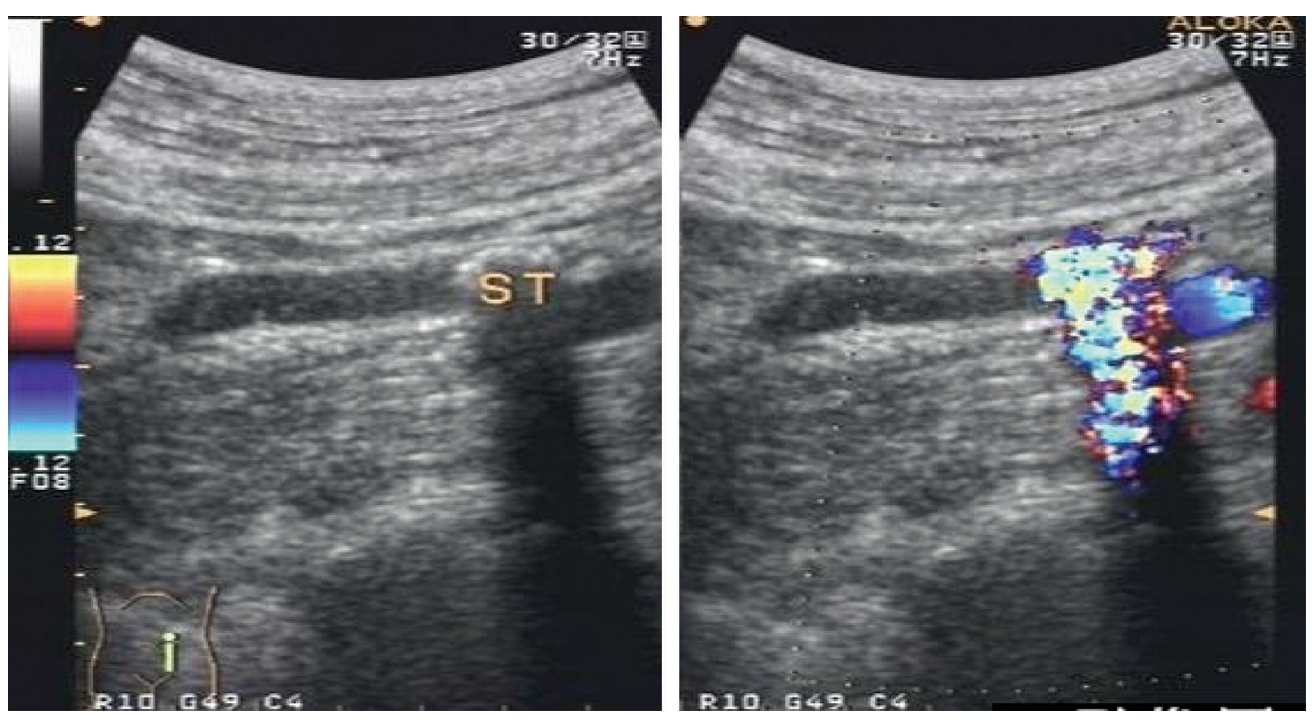

Figure 5: Ultrasound image of calculus in patient's ureter (male, 47 years old).

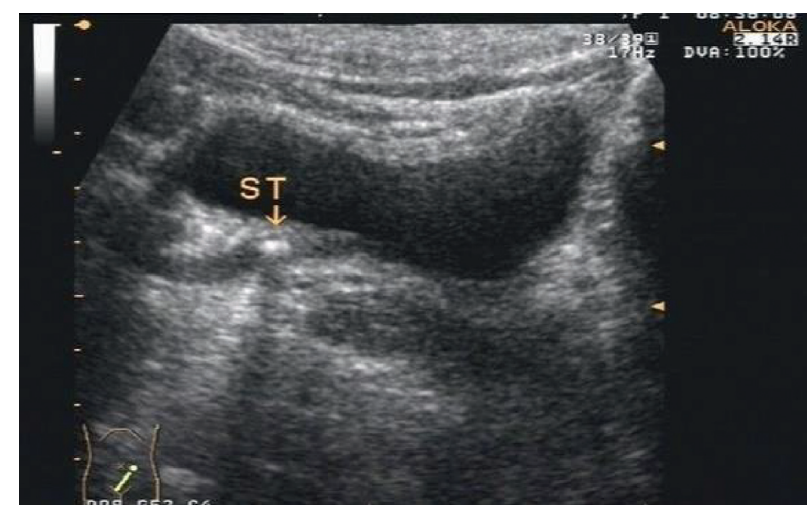

Figure 6: Ultrasound image of calculus in patient's ureter (female, 49 years old). 
control group, the number of successful operations in 46 patients was 44, and the success rate was over 95\% (Figure 7). The duration of operation for calculus in the observation group was $(29.7 \pm 7.65) \mathrm{min}$, while that in control group was $(40.7 \pm 8.36) \mathrm{min}$ (Figure 8$)$. The calculus excretion time of observation group was $(6.99 \pm 5.29)$ days, while that of control group was $(14.1 \pm 7.21)$ days (Figure 9). The length of hospital stay between the two groups was compared; the length of hospital stay of the observation group was $(3.67 \pm 2.9)$ days, and that of the control group was $(5.12 \pm 3.72)$ days (Figure 10). Based on the above data, the observation group was superior to the control group in terms of surgical success rate, operation duration, calculus clearance time, and length of hospital stay, with remarkable differences between the two $(P<0.05)$.

3.3. Comparison of Treatment Effects between the Two Groups. In Figure 11, the effective rate of the observation group was $97.7 \%$ and that of the control group was $84.3 \%$. The observation group was obviously superior to the control group, and the difference between the two groups was considerable $(P<0.05)$.

3.4. Comparison of Complications in the Two Groups. Among the complications of the two groups, the incidence of adverse reactions such as urinary tract infection, secondary hematuria, mucosal avulsion, and ureter perforation in the observation group was inferior to that in the control group. Both groups had complications after treatment. The complication rate in the observation group was $3.11 \%$, which was considerably lower than $19.1 \%$ in the control group. The difference between the two groups was great $(P<0.05)$ (Figure 12).

\section{Discussion}

Pneumatic lithotripsy first appeared in the 1990s as a novel type of intracavitary lithotripsy system. With continuous improvement and development, its utilization in various clinical fields has begun [15]. It is a safe, efficient, and affordable surgical method. In the treatment of urinary system disease, the adoption of pneumatic lithotripsy under flexible ureteroscope is more effective, and the damage to the ureteral mucosa is small, and the incidence of complications is also low. Holmium laser lithotripsy is a pulsed laser produced by holmium element [16]. The wavelength of holmium laser is in the absorption range of water, and surgery is also performed in a watery environment. When it irradiates local tissues, it will generate nearly kilowatt peak energy in an instant, which can cut and ablate local tissues. It has the characteristics of precise cutting, effective hemostasis, and small damage. Based on these physical and chemical properties, it can crush calculus of various compositions and densities [17]. The advantage of holmium laser lithotripsy is that it has high crushing efficiency, high speed, and ability to crush crushed stones of various compositions into $1 \sim 3 \mathrm{~mm}$

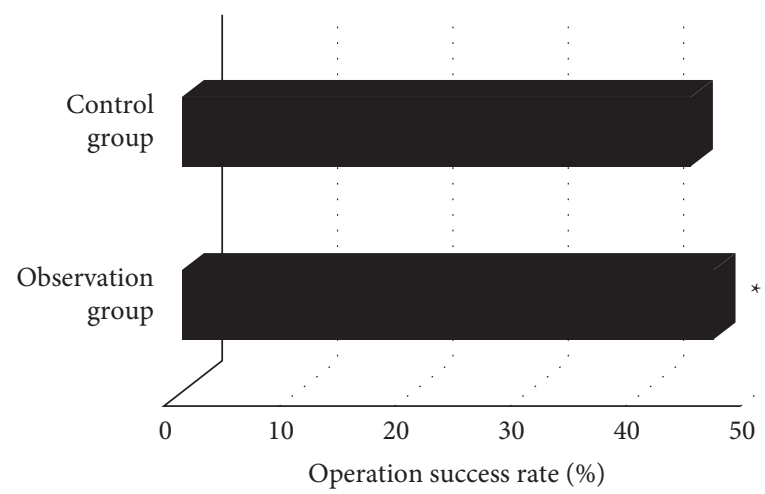

FIgURE 7: Comparison of the number of successful operations between the two groups $\left({ }^{*}\right.$ meant that the observation group was evidently different from the control group).

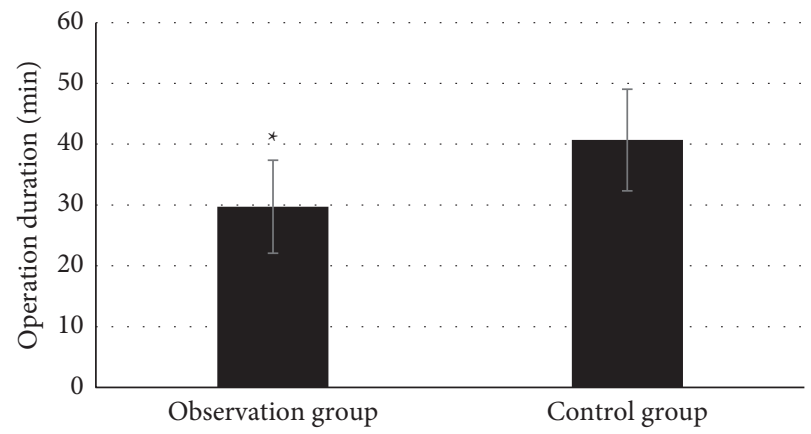

Figure 8: Comparison of operation duration between the two groups $\left({ }^{*}\right.$ meant that the observation group was evidently different from the control group).

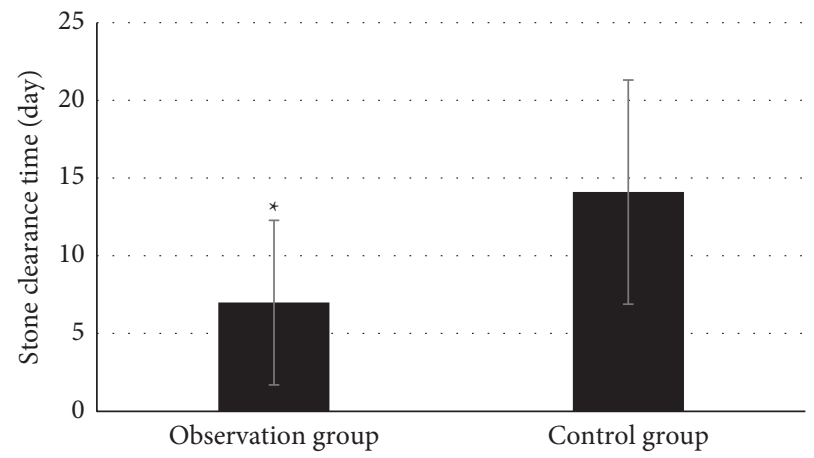

FIgURE 9: Comparison of calculus excretion time between the two groups $\left({ }^{*}\right.$ meant that the observation group was evidently different from the control group).

particles. In this way, calculus can be expelled from the patient's body quickly, and the success rate of lithotripsy of the operation is improved, especially for calculus in the upper ureter. The holmium laser can accurately cut and stop bleeding and can also deal with the problems of combined polyps, stenosis, and bleeding during the lithotripsy process. In the laser treatment adopted before, there were big shortcomings [18]. For example, nonpulsed carbon dioxide laser surgery's main principle is vaporizing calculus through the thermal effect. This surgical 


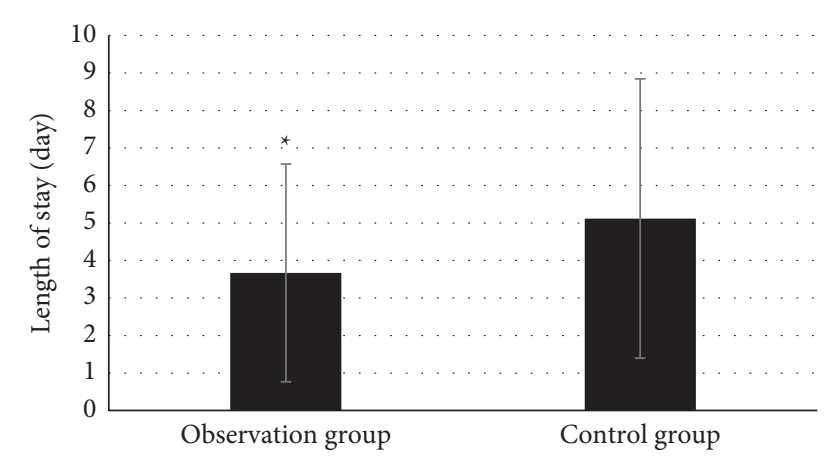

FIGURE 10: Comparison of length of hospital stay between two groups of patients $\left({ }^{*}\right.$ meant that the observation group was evidently different from the control group).

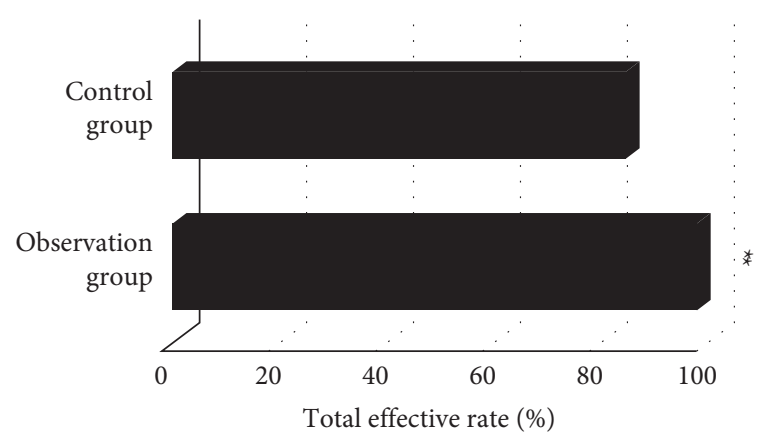

FIgURE 11: Comparison of the total effective rate of treatment between the two groups $\left({ }^{*}\right.$ meant that the observation group was evidently different from the control group).

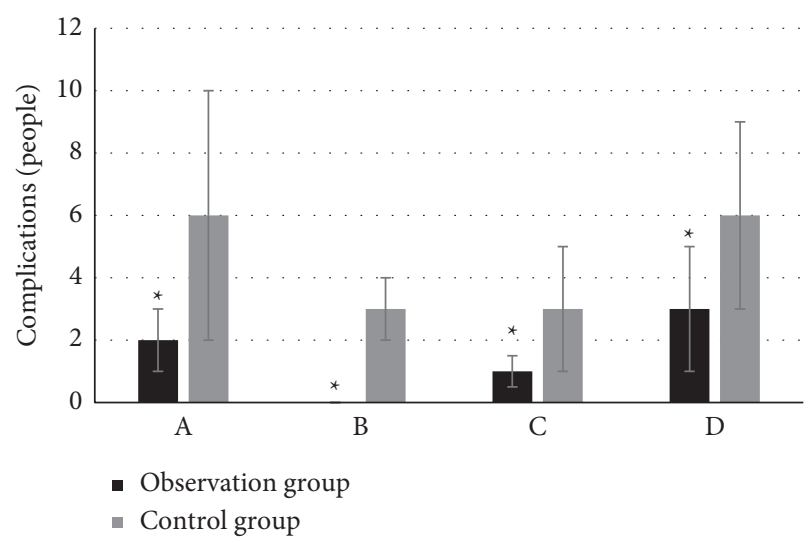

Figure 12: Comparison of postoperative complications between the two groups (A: urinary tract infection, B: secondary hematuria, C: mucosal avulsion, and D: ureteral perforation $)\left({ }^{*}\right.$ meant that the observation group was evidently different from the control group).

method will produce a higher temperature during the surgical treatment, up to $3000{ }^{\circ} \mathrm{C}$, which is extremely harmful to the patient's body. Moreover, the applicability of nonpulsed $\mathrm{CO}_{2}$ laser is also limited, which cannot crush acid calculus, and its accuracy is not ideal, so it is not recommended for clinical treatment [19].
For patients with urinary system examination, ultrasound technology is widely used, but there are many shortcomings such as blurred boundaries of pathological characteristics and many types of samples, which will affect the diagnosis of the disease. SVM is a classification method based on VC dimension theory and structural risk minimization principle dimension. According to the limited sample information, the best compromise between the complexity of the model (the learning accuracy of specific training samples) and the learning ability (the ability to identify any sample without error) is sought to obtain the best generalization ability. The technology of pattern recognition can be used in ultrasonic image segmentation, so it is widely used in medical image processing in recent years.

At present, the most commonly adopted clinical treatment of urinary system disease is surgical treatment. Traditional surgery for the treatment of UC involves dermatophytes, extracorporeal shock wave lithotripsy, and open surgery. However, these surgical methods will cause great surgical trauma to the patient's body, and the probability of postoperative complications is also high, such as postoperative bleeding, postoperative wound infection, and urine leakage. The adoption of holmium laser lithotripsy to treat UC can effectively increase the clearance rate of lithotripsy. Domestic studies revealed that utilizing holmium laser lithotripsy to treat calculus can have a single lithotripsy success rate as high as $93.7 \%$. Li et al. suggested that the combined soft ureteroscope combined with holmium laser treatment of kidney and upper ureter calculus can achieve 100\% lithotripsy clearance. Sun et al. found that in the treatment of holmium laser lithotripsy under flexible ureteroscopy, the success rate of lithotripsy in the upper calculus was higher than that of calculus in the lower calculus, and the safety was better. Winship et al. [20] studied the clinical efficacy of holmium laser lithotripsy, with 120 patients as the survey objects, and the maximum calculus size of the patient was $3.2 \mathrm{~cm}$. When holmium laser lithotripsy was utilized, the removal rate of a single operation using holmium laser lithotripsy was $63 \%$, and the secondary surgical clearance rate was $98 \%$.

In this research, holmium laser lithotripsy and pneumatic lithotripsy were employed to treat patients with UC. Both surgical methods had ideal lithotripsy clearance rate, and the lithotripsy clearance rate of holmium laser lithotripsy was more than $95 \%$, which can effectively crush the calculus in the patient and finally exclude it from the body, and it was also very safe. In the comparisons between groups, it was found that holmium laser lithotripsy had higher safety than pneumatic lithotripsy surgery. It can coagulate and stop bleeding while crushing calculus through pulsed laser. Under the action of the endoscope, the holmium laser can quickly find the specific position of the UC and directly crush the calculus. The results of this study were in line with expectations because holmium laser caused low physical damage to patients, which could largely promote the rapid rehabilitation of patients with stones and reduce the occurrence of complications. For the low resolution and low signal-to-noise ratio of ultrasonic images, the SVM algorithm used in this study can identify the clear part of the image and measure it, and the part with missing boundary 
was removed and not measured. It has high work efficiency. Holmium laser lithotripsy is currently widely adopted in clinical practice, and the treatment effect is more significant, which also has obvious advantages for calculus in other parts.

\section{Conclusion}

The postoperative treatment results of 92 UC patients were taken as a research sample. The results showed that after ultrasound image classification based on SVM was employed to select patient ultrasound images, under such auxiliary diagnosis and treatment, the speed and efficiency of diagnosis and treatment were effectively improved. The observation group that received holmium laser lithotripsy to treat UC was superior to the control group in terms of the success rate of surgery, the duration of surgery, the time of calculus discharge, the length of hospital stay, and the occurrence of postoperative complications. The test proved that the treatment of UC with holmium laser lithotripsy can effectively promote the recovery of urinary function in patients with UC. Moreover, it can effectively reduce the length of hospital stay, which had high clinical promotion value and significance.

The disadvantage of this study is that the sample size of selected patients is small and there is a certain deviation. The scope of adoption of the research results is also relatively small. In subsequent studies, the sample size of patients will be increased and the scope of the study will be expanded to further explore the clinical adoption value of holmium laser lithotripsy in various diseases. In short, the results provide a certain reference and significance for the treatment of UC patients.

\section{Data Availability}

The data used to support the findings of this study are available from the corresponding author upon request.

\section{Conflicts of Interest}

The authors declare that they have no conflicts of interest.

\section{Authors' Contributions}

$\mathrm{Hu} \mathrm{Li}$ and Zhijun Chen contributed equally to this work.

\section{References}

[1] J. Li, H. Yu, P. Zhou et al., "Application of flexible ureteroscopy combined with holmium laser lithotripsy and their therapeutic efficacy in the treatment of upper urinary stones in children and infants," Urology Journal, vol. 16, no. 4, pp. 343-346, 2019.

[2] E. X. Keller, V. de Coninck, M. Audouin et al., "Fragments and dust after Holmium laser lithotripsy with or without "Moses technology": how are they different?" Journal of Biophotonics, vol. 12, no. 4, Epub 2018 Dec 13. PMID: 30315636, Article ID e201800227, 2019.

[3] S. Chen, L. Zhou, T. Wei et al., "Comparison of holmium: YAG laser and pneumatic lithotripsy in the treatment of ureteral stones: an update meta-analysis," Urologia Internationalis, vol. 98, no. 2, pp. 125-133, 2017, Epub 2016 Aug 10. PMID: 27505176.

[4] Z. Adwin Zainal Abidin, F. Hayati, G. Hee Tan, E. Hong Goh, J. Jasman, and M. Zainuddin Zulkifli, "Giant urethral calculus without acute urinary retention," Journal of the College of Physicians and Surgeons Pakistan, vol. 28, no. 3, pp. S69-S70, 2018, PMID: 29482714.

[5] D. Habashy, R. Xia, W. Ridley, L. Chan, and L. Ridley, "Impact of dual energy characterization of urinary calculus on management," Journal of Medical Imaging and Radiation Oncology, vol. 60, no. 5, pp. 624-631, 2016, Epub 2016 Jul 28. PMID: 27469443.

[6] S. Cho, M. G. Park, K.-C. Lee, S. Y. Cho, and J. W. Lee, "Microbiological features and clinical factors associated with empirical antibiotic resistance in febrile patients with upper urinary tract calculi," Journal of Korean Medical Science, vol. 36, no. 1, p. e3, 2021 PMID: 33398940.

[7] U. G. Falagario, B. Calò, M. Auciello, G. Carrieri, and L. Cormio, "Advanced ureteroscopic techniques for the management of kidney stones," Current Opinion in Urology, vol. 31, no. 1, pp. 58-65, 2021, PMID: 33239516.

[8] Z. Fu, Z. Li, J. Huang et al., "High 18F-FDG uptake in urinary calculi on PET/CT: an unrecognized non-malignant accumulation," European Journal of Radiology, vol. 85, no. 8, pp. 1395-1399, 2016, Epub 2016 May 24. PMID: 27423678.

[9] A. Aggarwal, V. Singh, R. J. Sinha, and S. Pandey, "Large anterior urethral calculus masquerading as periurethral abscess," BMJ Case Reports, vol. 2018, PMID: 30413442, Article ID bcr2018225831, 2018.

[10] C. Wang, S. Lv, A. Wang et al., "A clinical study of choledochoscopic holmium laser lithotripsy for multiple intrahepatic calculi within ERAS programs," Lasers in Surgery and Medicine, vol. 51, no. 2, pp. 161-166, 2019, Epub 2018 Jul 19. PMID: 30024034.

[11] Z. Lv, L. Qiao, Q. Wang, and F. Piccialli, “Advanced machinelearning methods for brain-computer interfacing," IEEE/ ACM Transactions on Computational Biology and Bioinformatics, vol. 18, no. 5, pp. 1688-1698, 2021.

[12] A. H. Aldoukhi, T. L. Hall, K. R. Ghani, A. D. Maxwell, B. MacConaghy, and W. W. Roberts, "Caliceal fluid temperature during high-power holmium laser lithotripsy in an in vivo porcine model," Journal of Endourology, vol. 32, no. 8, pp. 724-729, 2018, Epub 2018 Jul 13. PMID: 29905092.

[13] V. S. C. Chernega, N. P. T.-S. Tlukpovskaya-Stepanenko, A. N. E. Eremenko, and S. N. E. Eremenko, "The assesment of the fragmentation rate of urinary stones during the holmium laser lithotripsy," Urologiia, vol. 5_2018, no. 5, pp. 69-72, 2018, Russian. PMID: 30575353.

[14] A. D. Maxwell, B. MacConaghy, J. D. Harper, A. H. Aldoukhi, T. L. Hall, and W. W. Roberts, "Simulation of laser lithotripsyinduced heating in the urinary tract," Journal of Endourology, vol. 33, no. 2, pp. 113-119, 2019, Epub 2019 Jan 29. PMID: 30585741 .

[15] Z. Wan, Y. Dong, Z. Yu, H. Lv, and Z. Lv, "Semi-supervised support vector machine for digital twins based brain image fusion," Frontiers in Neuroscience, vol. 15, Article ID 705323, 2021.

[16] M. M. Elhilali, S. Badaan, A. Ibrahim, and S. Andonian, "Use of the moses technology to improve holmium laser lithotripsy outcomes: a preclinical study," Journal of Endourology, vol. 31, no. 6, pp. 598-604, 2017, Epub 2017 Apr 25. PMID: 28340540. 
[17] S. Xie, Z. Yu, and Z. Lv, "Multi-disease prediction based on deep learning: a survey," Computer Modeling in Engineering \& Sciences, vol. 128, no. 2, pp. 489-522, 2021.

[18] R. Li, D. Ruckle, M. Keheila et al., "High-frequency dusting versus conventional holmium laser lithotripsy for intrarenal and ureteral calculi," Journal of Endourology, vol. 31, no. 3, pp. 272-277, 2017, Epub 2017 Jan 16. PMID: 27960541.

[19] C. Esposito, L. Masieri, V. Bagnara, B. Tokar, A. Golebiewski, and M. Escolino, "Ureteroscopic lithotripsy for ureteral stones in children using holmium: yag laser energy: results of a multicentric survey," Journal of Pediatric Urology, vol. 15, no. 4, 391 pages, 2019, Epub 2019 May 8. PMID: 31182399.

[20] B. Winship, D. Wollin, E. Carlos et al., "The rise and fall of high temperatures during ureteroscopic holmium laser lithotripsy," Journal of Endourology, vol. 33, no. 10, pp. 794-799, 2019, Epub 2019 May 27. PMID: 31016991. 